\title{
In Vitro Proinflammatory Polarization of Macrophages Isolated from Hepatocarcinogenic Stage in Humans and Rats
}

\author{
MARTA WÓJCIK ${ }^{1}$, JOANNA WESSELY-SZPONDER ${ }^{1}$, HALINA CICHOŻ-LACH ${ }^{2}$, \\ KRZYSZTOF CELIŃSKI ${ }^{2}$ and RYSZARD BOBOWIEC ${ }^{1}$ \\ ${ }^{1}$ Department of Pathophysiology, Faculty of Veterinary Medicine, \\ University of Life Sciences in Lublin, Lublin, Poland; \\ ${ }^{2}$ Department of Gastroenterology with Endoscopic Unit, II Faculty of Medicine with English Language Division, \\ Medical University in Lublin, Lublin, Poland
}

\begin{abstract}
Macrophages have multiple functions in both inhibiting and promoting hepatocarcinogenesis, which are dependent on their phenotypes. Thus, we were interested in clarifying the 'training' proinflammatory effects exerted by barley $\beta$-glucan (BBG) on monocyte-derived macrophages from patients with hepatocellular carcinoma (HCC-Mfs). After isolation and differentiation HCC-Mfs were treated with different concentrations of $B B G$ and functional assays were then conducted after $24 h$, and 3 and 5 days of incubation. The release of reactive oxygen species, arginase concentration and cell morphology were analyzed. Under the influence of BBG neoplastic cells slightly elongated and dendric-like filopodia were observed. In HCC-Mfs, the significant generation of $\mathrm{NO}$ and $\mathrm{O}_{2}^{-}$. was seen on days 3 and 5 of culture, concomitantly with significant depletion $(p<0.05)$ of arginase activity. In summary, we showed that HCC-Mfs, 'trained' in a BBG microenvironment keep a highly dynamic plasticity, together with their proinflammatory polarization, expressed by reactive oxygen species and reactive nitrogen intermediates (RNI) augmentation.
\end{abstract}

Differently from irreversible phenotypic changes seen in lymphocytes after exposure to polarizing cytokines, macrophage polarization is transient and plastic. In order to adapt to the microenvironmental conditions of surrounding tissues, macrophages are able to rapidly switch their phenotypes. Therefore, exploring the dynamic process of macrophage polarization and the mechanisms that govern this process is not only important for our understanding of

Correspondence to: Dr Marta Wójcik, Akademicka 12, 20-033 Lublin, Poland. E-mail: marta.wojcik@up.lublin.pl

Key Words: Macrophages, $\beta$-glucan, trained immunity, hepatocellular carcinoma. macrophage polarization but may also provide new therapeutic strategies for various diseases including cancer (1). Hepatocellular carcinoma (HCC) is currently the fifth most common solid tumor worldwide. It is also the fourth leading cause of cancer-related deaths (2). Most patients with HCC suffer from coexisting cirrhosis, which is the major risk factor, and correlated with hepatitis $\mathrm{B}$ or $\mathrm{C}$ virus infection (2). Diethylnitrosamine (DEN) is an experimental hepatocarcinogen found in a variety of products to which humans may also be exposed, including tobacco smoke, meat, and whiskey $(3,4)$. Although much is known about the etiological agents of HCC, the cellular and molecular pathogenesis is not well understood. Moreover the effective therapy of this cancer is not exist and only palliative treatments can be offered (5).

In the tumor microenvironment, innate immune cells, represented by macrophages can be polarized by many stimuli. Two distinct polarization states have been described for macrophages: the M1 classically activated and M2 alternatively activated macrophage. The M1 phenotype is proinflammatory and is characterized by the release of inflammatory cytokines, reactive nitrogen intermediates (RNI), reactive oxygen species (ROS) and microbiocidal/ tumoridical activity (6). In contrast, M2 macrophages have an immunosuppressive and tumor-promoting phenotype. One novel strategy during tumor therapy is to prevent M2 polarization or reorientate M2-derived tumor-associated macrophages to the M1 phenotype.

Using an in vitro approach, some authors demonstrated that short priming of monocytes with $\beta$-glucan (contain mixedlinkages with 1,3- and 1,4 $\beta$-D-glucopyranose polymers) induces trained immune cells that are characterized by an enhanced inflammatory status $(7,8)$. Moreover, barley-derived $\beta$-glucan (BBG) binds to dectin-1 to exert immunostimulatory effects and might be applied in immunotherapy as a potent nontoxic immunostimulator (8). Thus, in our preliminary experiment we assessed the 'training' effects exerted by BBG 


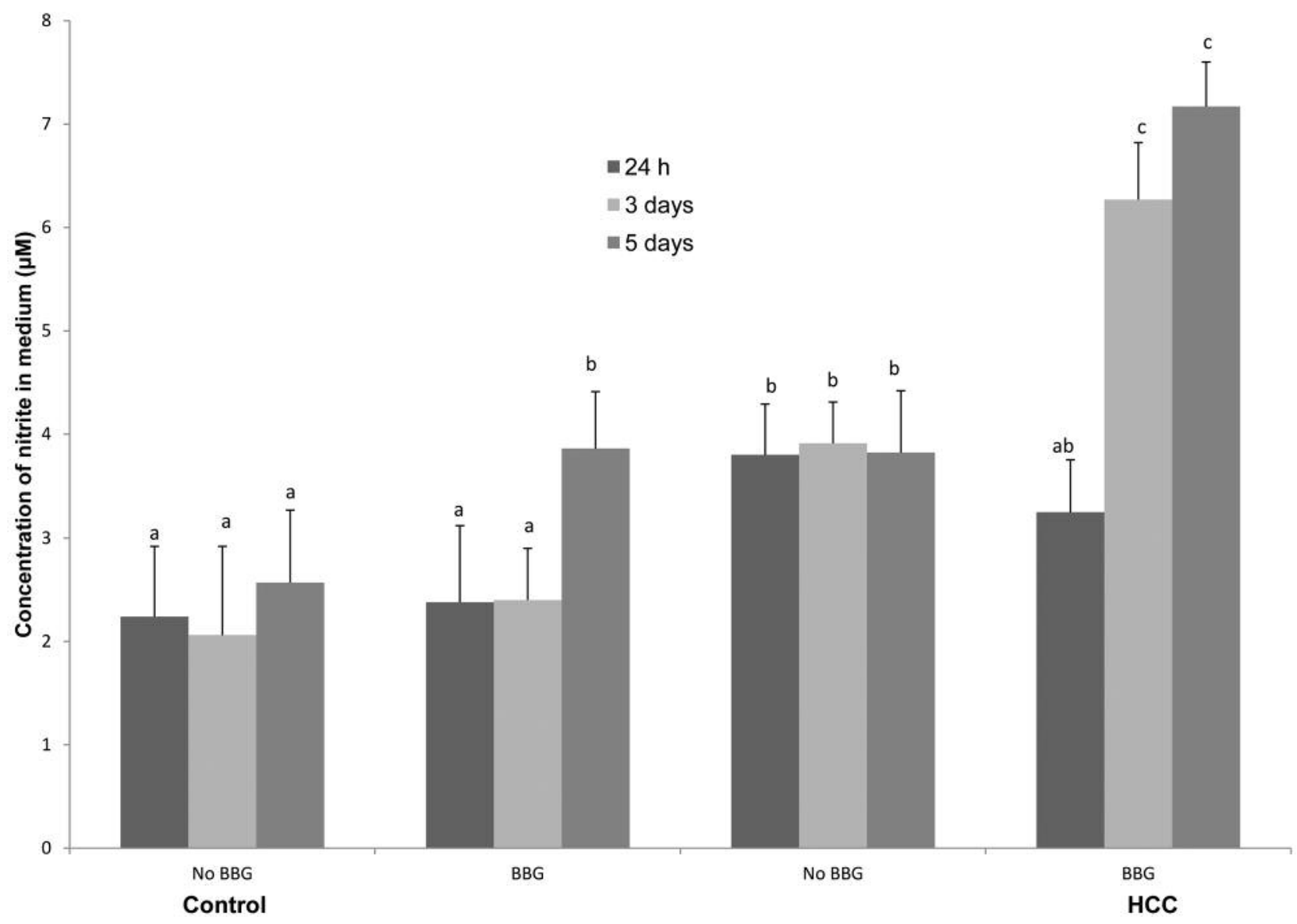

Figure 1. Concentration of nitrite in medium from cultures of unstimulated $(0 \mu \mathrm{g} / \mathrm{ml}$ barley beta glucan-(BBG) or stimulated (10 $\mu \mathrm{g} / \mathrm{ml} B B G)$ macrophages from healthy individuals and patients with hepatocellular carcinoma (HCC) after $24 \mathrm{~h}, 3$ days and 5 days of culture. Results are expressed as the mean $\pm S D$ obtained from each individual experiment. Mean values marked with different letters differ statistically $(p<0.05)$. Comparisons were made using an ANOVA test.

on monocyte-derived macrophages in vitro under control and neoplastic conditions. In order to obtain a full representation of our findings, the study was conducted on macrophages obtained from cells isolated from blood of healthy individuals and patients with HCC, and then compared with those from a rat DEN-induced model of neoplasia.

\section{Materials and Methods}

Human monocyte isolation. The study was approved by the Local Bioethics Committee (no. KE-0254/329/2015) and participants gave their written informed consent. Six patients with HCC and six without HCC had been randomly selected from patients admitted to the Gastroenterology Clinic of Public University Hospital No 4 in Lublin from Jan to May 2016. The patients with HCC patients were classified with class B disease (7-9 points), based on the Child-Pugh score. Human peripheral blood from healthy male donors and patients with HCC was drawn by venapuncture in EDTA-coated tubes. The mononuclear cell fraction was isolated by densitygradient centrifugation using Lymphoprep (Nyegaard \& Co, AS,
Oslo, Norway). The number and viability of obtained cells were determined using R1 Automated Cell Counter (Olympus, Warsaw, Poland), and then cells were plated into wells of a 96 -well plate at a density of $1.0 \times 10^{6}$ cells $/ \mathrm{ml}$.

Animals and experimental design. Ten-week-old female Wistar rats (Medical University in Białystok, Poland) weighing 200-250 g were used in this study. Animals were kept in a temperature- and humidity-controlled room with a 12-h light-dark cycle.

After 1-week period of acclimatization, rats were divided into two groups: I: control group $(n=5)$ fed with standard diet; and II: neoplastic group $(n=5)$. For the second group, for HCC induction, after partial hepatectomy, genotoxic DEN (Sigma Aldrich, Poznan, Poland) was given. Partial (two-third) hepatectomy was performed according to the Higgins and Anderson method by excision of left lateral and right lobes (2/3 partial hepatectomy) (4). All surgical procedures were carried out under light anesthesia between 9:00 AM to 12:00 AM to minimize the diurnal effect of liver regeneration. Diethylnitrosamine was applied at $0.005 \%$ in drinking water for 6 weeks. All procedures were approved by the local Ethical Commission (decision number 81/2015). 


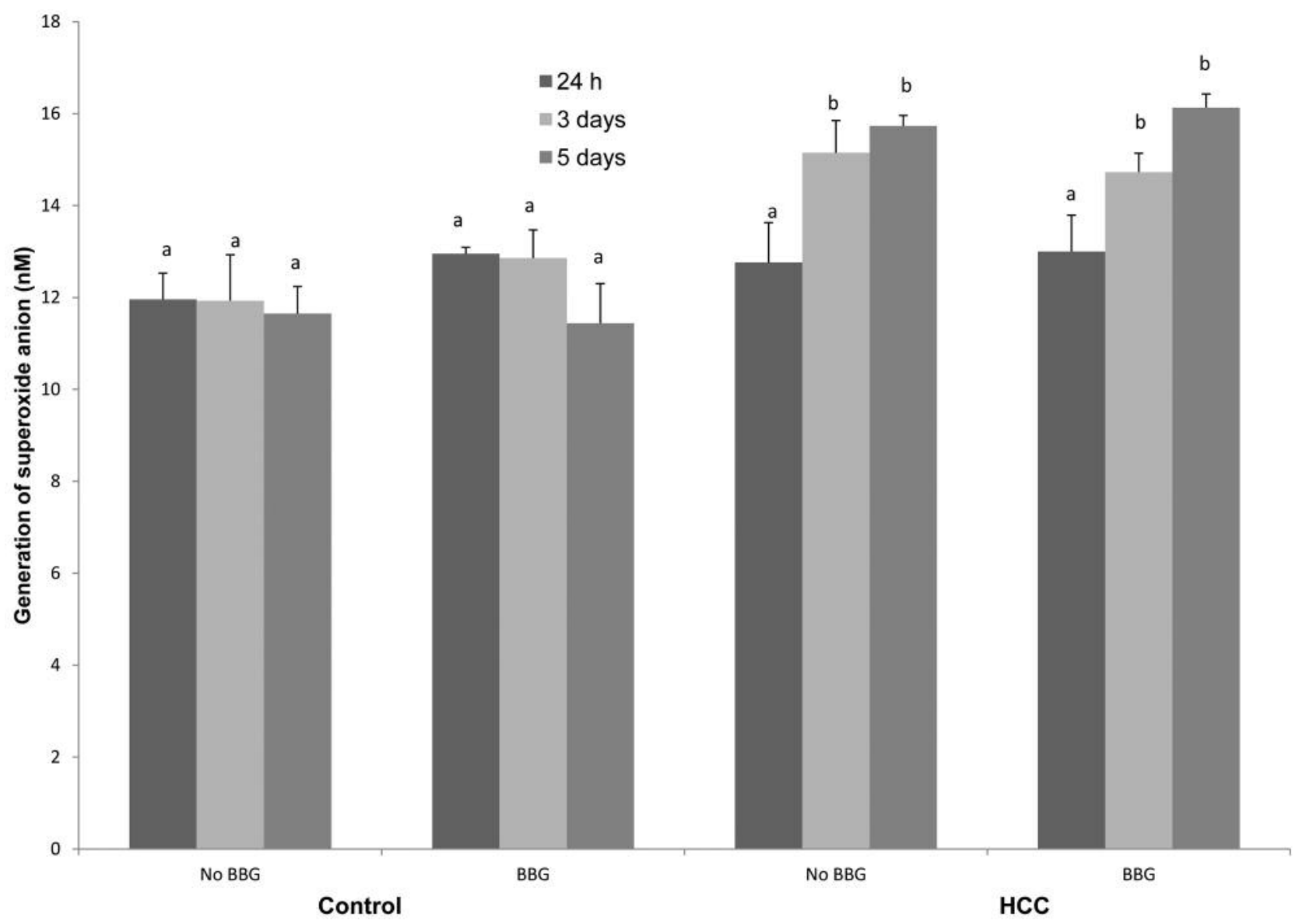

Figure 2. Generation of superoxide anion in medium from cultures of unstimulated ( $0 \mu \mathrm{g} / \mathrm{ml} B B G$ ) or stimulated (10 $\mu \mathrm{g} / \mathrm{ml} \mathrm{BBG)} \mathrm{macrophages} \mathrm{from}$ healthy individuals and patients with HCC after $24 \mathrm{~h}, 3$ days and 5 days of culture. Values are means \pm SD obtained from each separate experiment. Mean values marked with different letters differ statistically $(p<0.05)$.

After 6 weeks of DEN administration, hepatocytes were isolated from both groups of animals as described previously, with minor modifications (4). Before laparotomy, animals were anesthetized with intramuscular administration of a mixture of ketamine $(90 \mathrm{mg} / \mathrm{kg}$ bwt $)$ and xylazine $(10 \mathrm{mg} / \mathrm{kg}$ bwt). The liver was perfused in situ through the portal vein by a Krebs-Ringer buffer: a) containing EGTA; b) without $\mathrm{Ca}^{2+}$ and chelating agent; or c) with type IV collagenase.

Isolation of rat blood-derived monocytes. Blood was taken from each animal during the experimental procedure but before liver perfusion. The obtained blood was collected into a heparinized syringe then mononuclear cells were isolated using Lymphoprep density-gradient centrifugation. Cell count and viability were assessed using R1 Automated Cell Counter (Olympus) and viability was found to be $>80 \%$. After being counted, cells were plated into wells of a 96-well plate at a density of $1.0 \times 10^{6}$ cells $/ \mathrm{ml}$ and cultured at $37^{\circ} \mathrm{C}$ with $5 \% \mathrm{CO}_{2}$ for $24 \mathrm{~h}$ in Dulbecco's modified Eagle's medium with $10 \%$ calf serum. After incubation, the medium containing non-adherent cells was removed by aspiration and the plate was washed to obtain adherent monocytes (9).
Human and rat macrophage differentiation. The adherent cells were cultured for an additional $48 \mathrm{~h}$ to allow monocytes to mature into functional macrophages. After differentiation, human macrophages from both groups, healthy individuals and patients with HCC, were treated with BBG (Sigma-Aldrich, Poznan, Poland) at concentration of $10 \mu \mathrm{g} / \mathrm{ml}$ or with phosphate buffered saline (PBS; Biomed, Lublin, Poland) in equal volumes and incubated for $24 \mathrm{~h}$. Functional assays were then conducted and repeated after 3 and 5 days of incubation (10).

Rat macrophages after differentiation were stimulated with BBG at concentrations of $5,10,20 \mu \mathrm{g} / \mathrm{ml}$ and incubated for $24 \mathrm{~h}$. All functional assays were repeated after 3 and 5 days of incubation. The control used macrophages exposed to culture medium containing PBS in a similar amount as other treatments.

Functional assays of blood monocyte-derived macrophages.

Nitrite determination: The accumulation of nitrite in the culture supernatant of cells after treatment with different concentrations of BBG for $24 \mathrm{~h}$ was measured as described previously (11). Here, $100 \mu \mathrm{l}$ aliquots of the culture supernatant were mixed with an equal volume of Griess reagent (1:1 mixture of naphtylethylenediamine, 


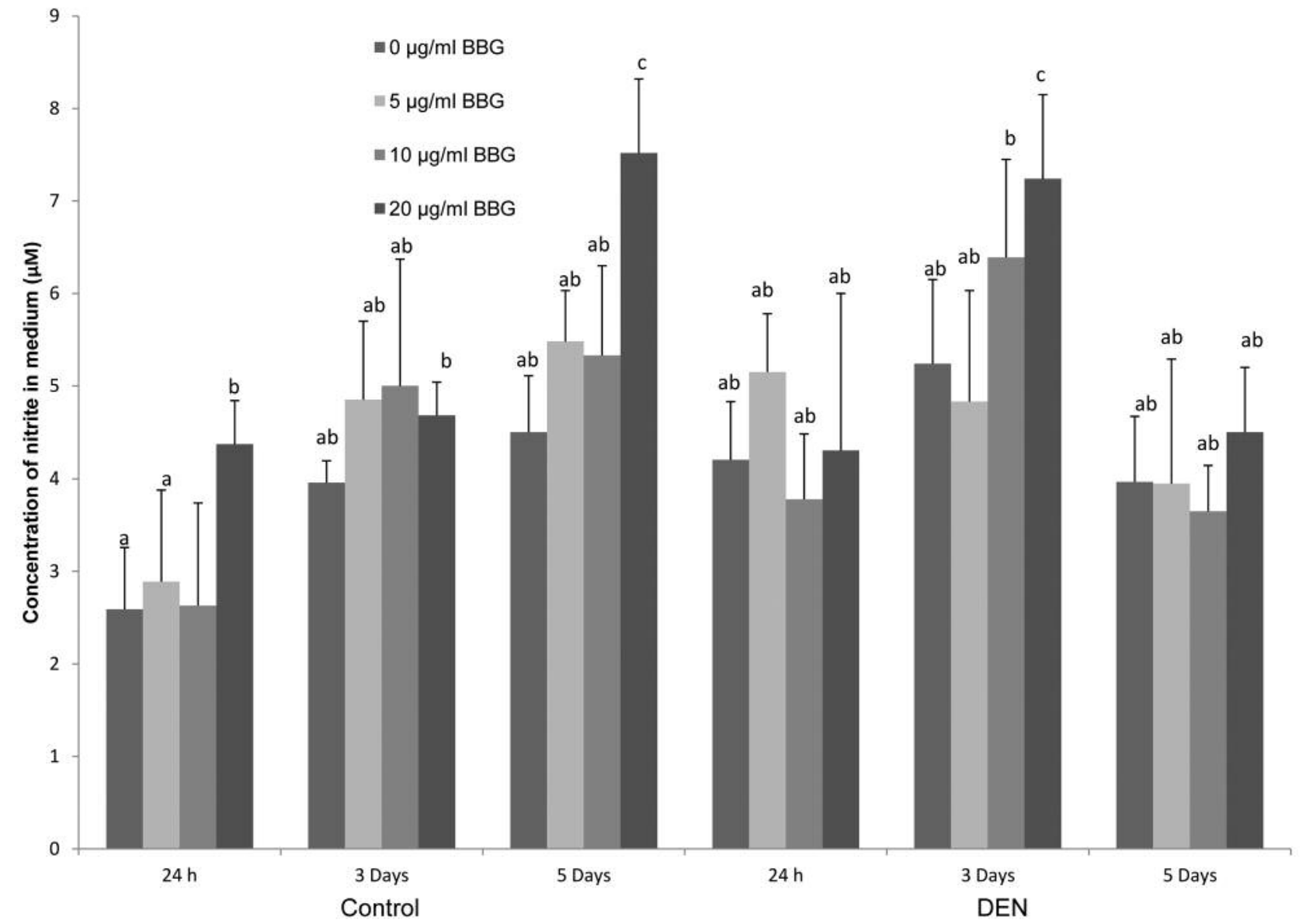

Figure 3. Concentration of nitrite in medium from cultures of unstimulated $(0 \mu \mathrm{g} / \mathrm{ml} \mathrm{BBG})$ or stimulated $(5,10 \mathrm{or} 20 \mu \mathrm{g} / \mathrm{ml} \mathrm{BBG}) \mathrm{microphages}$ from control and DEN-treated rats after $24 \mathrm{~h}, 3$ days and 5 days of culture. Values are means $\pm S D$ obtained from each separate experiment. Mean values marked with different letters differ statistically $(p<0.05)$.

dihydrochloride and $1 \%$ sulfanilamide in $5 \% \mathrm{H}_{3} \mathrm{PO}_{4}$ ) and incubated at room temperature for $15 \mathrm{~min}$. The nitrite concentration was calculated from a $\mathrm{NaNO}_{2}$ standard curve.

Superoxide anion generation: Superoxide anion generation was measured by a nitrotetrazolium (NBT) reduction assay as described previously (11). Briefly, cultures were incubated with $0.1 \%$ nitroblue tetrazolium (NBT; Sigma) solution at room temperature for $15 \mathrm{~min}$ and then the absorbance was read. Nanomoles of superoxide produced over the incubation period were calculated using the extinction coefficient of NBT $(21.1 \mathrm{nMol})$.

Arginase assay: Arginase activity was assessed by the measuring the concentration of urea generated by the arginase-dependent hydrolysis of L-arginine. After 5 days of culture, macrophages were lyzed with $50 \mu \mathrm{l}$ of $0.1 \%$ Triton X-100. After 30 min of incubation, $50 \mu \mathrm{l}$ of $25 \mathrm{mM}$ Tris- $\mathrm{HCl}$ and $10 \mu \mathrm{l}$ of $10 \mathrm{mM} \mathrm{MnCl}$, were added, and arginase was activated by heating for $10 \mathrm{~min}$ at $55^{\circ} \mathrm{C} .100 \mu \mathrm{l}$ of L-Arginine $(0.5 \mathrm{M})$ was hydrolyzed at $37^{\circ} \mathrm{C}$ for $120 \mathrm{~min}$. The reaction was then stopped with $400 \mu$ of $\mathrm{H}_{2} \mathrm{SO}_{4} / \mathrm{H}_{3} \mathrm{PO}_{4} / \mathrm{H}_{2} \mathrm{O}(1 / 3 / 7$ $\mathrm{v} / \mathrm{v} / \mathrm{v})$. The urea concentration was measured after the addition of $40 \mu \mathrm{l}$ of $\alpha$-isonitrosopropiophenone (Sigma-Aldrich) followed by heating at $100^{\circ} \mathrm{C}$ for $40 \mathrm{~min}$. The concentration of urea was determined by comparison with a standard curve of 1 to $100 \mu \mathrm{g} / \mathrm{ml}$ urea (12).

3-(4,5-dimethylthiazol-2yl)-diphenyltetrazolium bromide (MTT) assay. Cell respiration, an indicator of cell viability, was assessed by the mitochondrial dependent reduction of MTT to formazan. Briefly, $10 \mu \mathrm{l}$ of MTT solution was added to each culture. After incubation at $37^{\circ} \mathrm{C}$ with $5 \% \mathrm{CO}_{2}$ for $3 \mathrm{~h}, 100 \mu \mathrm{l}$ of $10 \%$ sodium dodecyl sulphate (Sigma-Aldrich) was added and cells were further incubated for $24 \mathrm{~h}$. The amount of formazan was then determined spectrometrically (13).

Morphological characterization. Every subsequent day of culture, macrophages were subjected to microscopic analysis of their morphology, as a qualitative assay.

Statistical analysis. The examined values were compared using Microsoft Excel and STATISTICA.PL analysis software. Results were expressed as the mean $\pm \mathrm{SD}$, and the data obtained were 


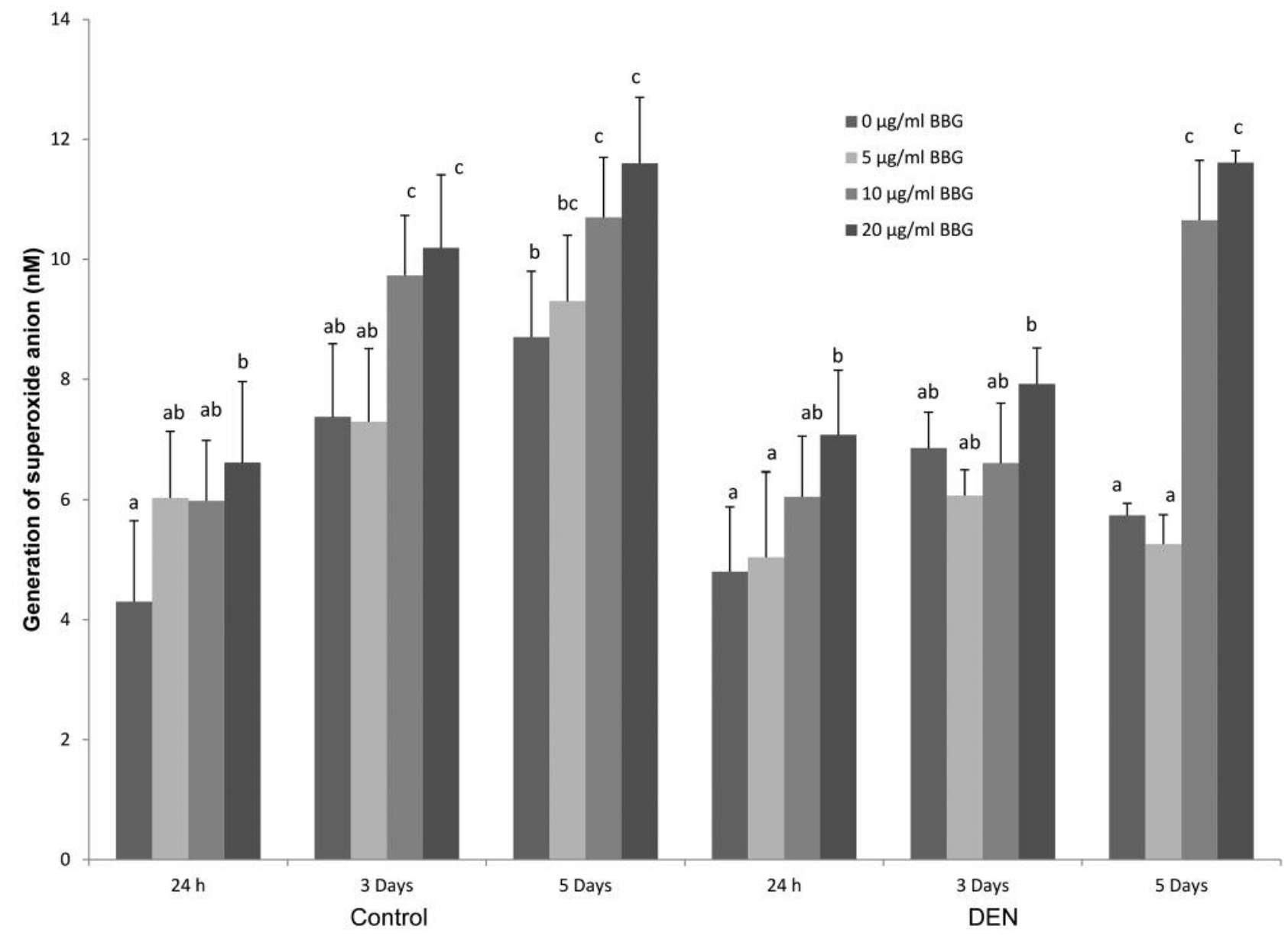

Figure 4. Generation of superoxide anion in medium from cultures of unstimulated $(0 \mu \mathrm{g} / \mathrm{ml}$ BBG) or stimulated $(5,10$ or $20 \mu \mathrm{g} / \mathrm{ml} B B G)$ microphages from control and DEN-treated rats after $24 \mathrm{~h}, 3$ days and 5 days of culture. Values are means \pm SD obtained from each separated experiment. Mean values marked with different letters differ statistically $(p<0.05)$.

evaluated by ANOVA and Student's $t$-test as appropriate. The level of significance was set at $p<0.05$.

\section{Results}

Free radical generation by human macrophages. A significant $(p<0.05)$ increase of nitric oxide generation was observed in macrophages obtained from healthy humans after 5 days of culture under the influence of $10 \mu \mathrm{g} / \mathrm{ml}$ of BBG. In macrophages from patients with $\mathrm{HCC}$, a significant response to $10 \mu \mathrm{g} / \mathrm{ml}$ of $\mathrm{BBG}$ was seen at 3 and 5 day of culture and was higher than that of macrophages from healthy individuals. Changes in unstimulated cultures from both patients and healthy donors were insignificant throughout the period of culture (Figure 1).

We did not observe any significant response to BBG in respect to superoxide anion generation in cultures of macrophages derived from healthy humans. In HCC-derived macrophages, there was a significant increase of $\mathrm{NO}$ production after 3 and 5 days of culture, however, without significant differences between stimulated and unstimulated cultures (Figure 2).

Free radical generation by rat macrophages. Because of poor response to $10 \mu \mathrm{g} / \mathrm{ml}$ of $\mathrm{BBG}$, we decided to use additional concentrations 5 and $20 \mu \mathrm{g} / \mathrm{ml}$ of BGG to stimulate rat macrophages. A significant increase of NO concentration in comparison with unstimulated cells in cultures of macrophages from control rats was observed in all measurements at $20 \mu \mathrm{g} / \mathrm{ml}$. On the other hand, a significant response to $\mathrm{BBG}$ in cells from DEN-treated rats was noted only on day 3 of incubation at $20 \mu \mathrm{g} / \mathrm{ml}$ (Figure 3 ).

In cultures of macrophages from control rats, a marked $(p \leq 0.05)$ response to BBG was detected only at maximal concentration of $20 \mu \mathrm{g} / \mathrm{ml}$ at the first measurement (after $24 \mathrm{~h}$ ), and at 10 and $20 \mu \mathrm{g} / \mathrm{ml}$ in at 3 and 5 days of culture. In 


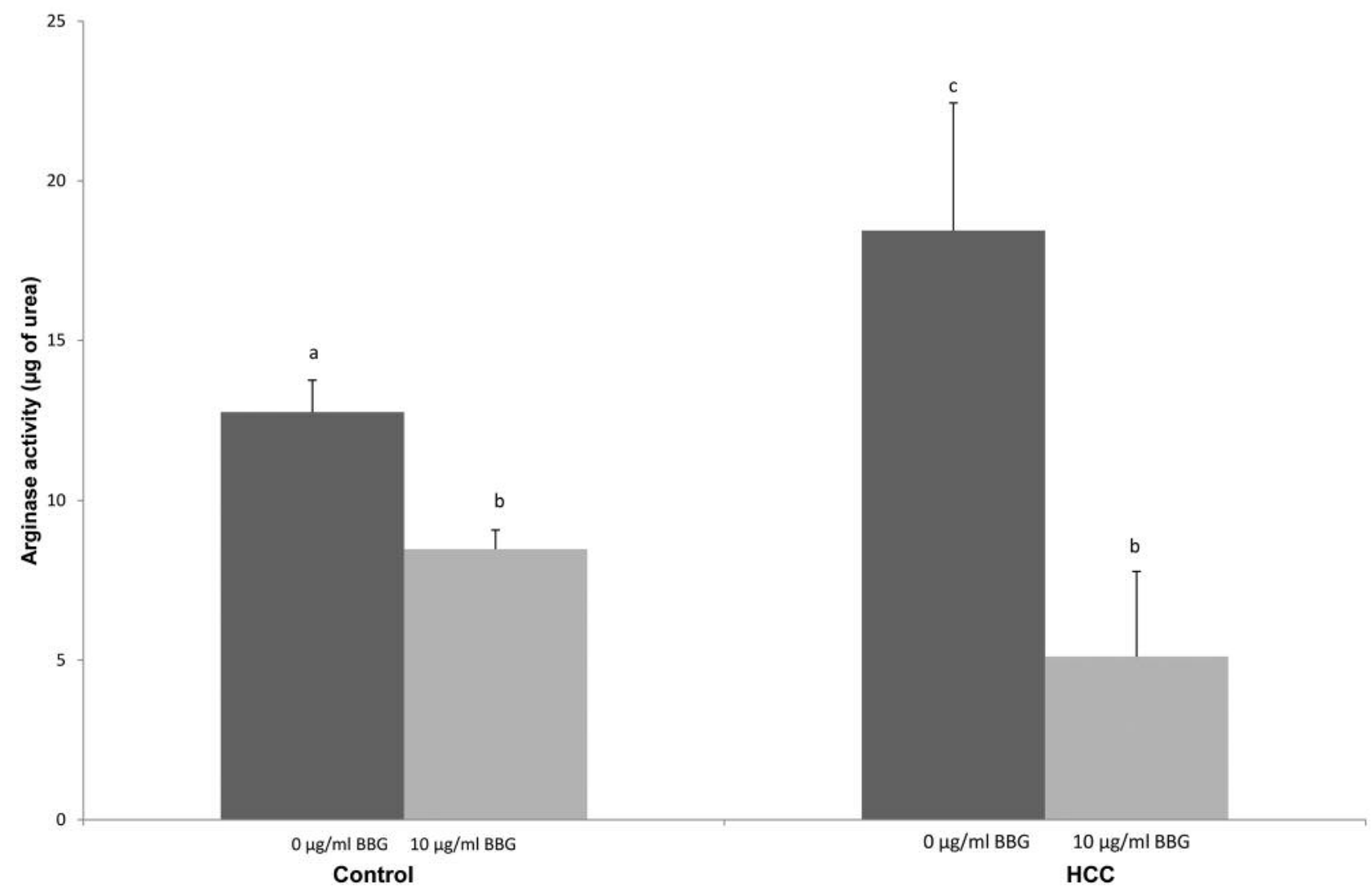

Figure 5. Concentration of urea as a marker of arginase activity of unstimulated ( $0 \mu \mathrm{g} / \mathrm{ml} \mathrm{BBG}$ ) or stimulated (10 $\mu \mathrm{g} / \mathrm{ml}$ BBG) macrophages from healthy individuals and patients with HCC after 5 days of culture. Values are means \pm SD obtained from each separated experiment. Mean values marked with different letters differ statistically $(p<0.05)$.

macrophages from DEN-treated rats, after $24 \mathrm{~h}$, generation of superoxide had only significantly increased under the influence of $20 \mu \mathrm{g} / \mathrm{ml}$ of BBG (Figure 4). A similar response in macrophages from DEN-treated rats was observed at the second time point, whereas on day 5 of culture there was a marked increase of generation of superoxide anion under the influence of $10 \mu \mathrm{g}$ as well as $20 \mu \mathrm{g}$ of BBG.

MTT assay. BBG did not significantly diminish cell respiration at the concentrations used $(5-20 \mu \mathrm{g} / \mathrm{ml})$ (data not shown).

Arginase activity assay. For human macrophages, arginase activity was higher in unstimulated cultures, whereas under the influence of BBG, activity of arginase decreased significantly $(p<0.05)$ in both control and HCC groups (Figure 5). In cultures of macrophages from healthy rats, the changes in arginase activity were insignificant, whereas in macrophages from DENtreated rats, a significant decrease in arginase activity was seen under the influence of $5 \mu \mathrm{g} / \mathrm{ml}$ of BBG (Figure 6).

In order to evaluate the relationship between pro- and antiinflammatory immune response, we calculated the ratio of urea to nitrite production. This ratio was significantly enhanced under the influence of BBG in macrophages from patients with HCC, and was higher in DEN-treated rats in comparison with the control rat group (Figure 7).

Morphological changes. Morphological changes of monocytes and macrophages are shown in Figure 8. Under the influence of BBG, cells were slightly elongated and dendric-like filopodia were observed. In the case of HCC cultures, activation of macrophages was visible in both cultures stimulated with BBG and unstimulated ones after 5 days of culture.

Unstimulated rat macrophages were rounded, whereas under the influence of BBG activation, cells generated long filopodia, especially in cells from DEN-treated rats after 5 days of culture at all concentrations of BBG.

\section{Discussion}

In our experiment, release of NO by macrophages from patients with $\mathrm{HCC}$ without stimulation was lower than that by macrophages treated with BBG. 


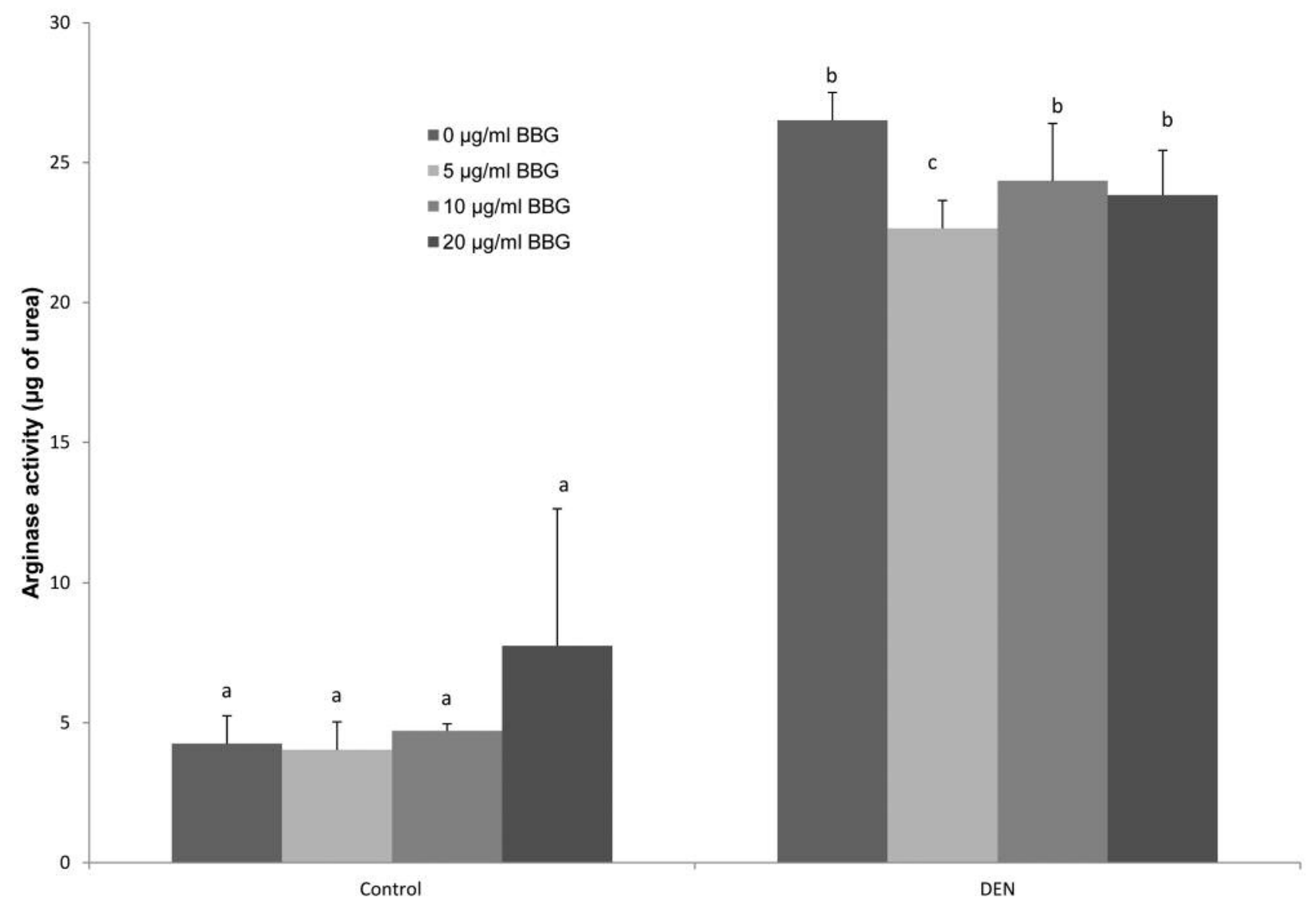

Figure 6. Concentration of urea as a marker of arginase activity of $M f s$ unstimulated $(0 \mu \mathrm{g} / \mathrm{ml} B B G)$ unstimulated $(0 \mu \mathrm{g} / \mathrm{ml}$ BBG) or stimulated (with 5,10 or $20 \mu \mathrm{g} / \mathrm{ml} \mathrm{BBG}$ ) from control and DEN rats after 5 days of culture. Values are means $\pm S D$ obtained from each separated experiment. Mean values marked with different letters differ statistically $(p<0.05)$.

As estimated previously, classically activated M1 macrophages up-regulate expression of pro-inflammatory cytokines, including TNF $\alpha$, IL6 and interferon- $\gamma$ and they increase the production of ROS and nitrogen intermediates (RNI) $(3,6)$. M1 macrophages express inducible NO synthase (iNOS) and metabolize arginine by releasing NOR (or RNI) and citruline, whereas M2 macrophages up-regulate arginase (I and II) to metabolize arginine into urea and L-ornithine (3). In rat macrophages, in turn, increase of $\mathrm{NO}$ generation was noted in control animals at a dose of $20 \mu \mathrm{g} / \mathrm{ml}$ of $\beta$-glucan, whereas in DEN-treated rats the response was shown only on the third day under the influence of maximal dose of $\beta$-glucan. Previously, glucan was evaluated for its ability to modify the hepatic and renal tumorigenesis induced in partially hepatectomized Sprague-Dawley female rats by DEN. The results indicated that glucan did not significantly modify the incidence of the chemically-induced hepatic tumors (14). Michałek et al. revealed that poly-[1-6]-D-glucopyranosyl-[13]-D-glucopyranose (PGG)-glucan activated rat macrophages but they did not study generation of NO by these cells (15).

We observed a decrease of arginase activity in human macrophages from both healthy and $\mathrm{HCC}$ groups stimulated with BBG. According to Geelhaar-Karsch et al., arginase activity does not reflect the differentiation state of human macrophages, but seems to be a reasonable functional test to detect enhanced M2 macrophage activation (16). In the case of rat macrophages, we noted a significant decrease of arginase activity only in macrophages from DEN-treated rats under the influence of $5 \mu \mathrm{g}$ of BBG.

We found significant differences only in macrophages from patients with $\mathrm{HCC}$, where in unstimulated culture the ratio was significantly higher than in cultures stimulated with BBG. In DEN-treated rats, in turn, both ratios in stimulated and unstimulated cultures were higher than in those with human macrophages but without differences between groups studied. M2 polarized macrophages express a high level of arginase that competes with iNOS for L-arginine, the common substrate of both, suppressing the production of NO 


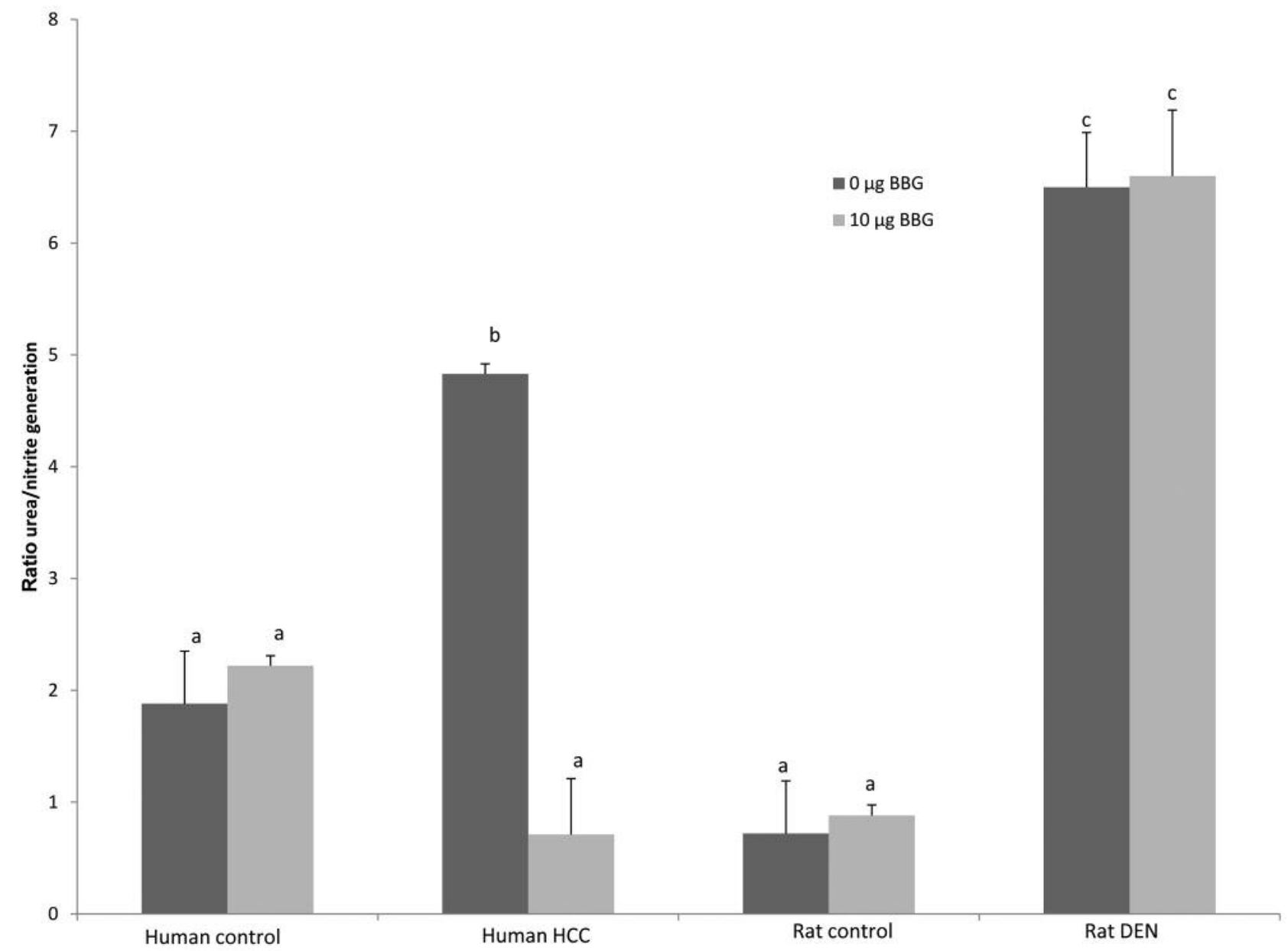

Figure 7. Ratio between urea and nitrite generation of macrophages from rats and humans unstimulated or under the influence of $10 \mu \mathrm{g} / \mathrm{ml}$ of $B B G$. Values are means $\pm S D$ obtained from each separate experiment. Mean values marked with different letters differ statistically $(p<0.05)$.

and converting L-arginine into urea (17). Indeed, the balance between iNOS and arginase activity is tightly regulated during repair (18). Geelhaar-Karsh et al. considered the urea/nitrite ratio as a marker of differentiation of human macrophages, however, this has not yet been considered in tumor progression (16).

We did not observe any significant response to BBG with respect to superoxide anion generation in cultures of macrophages derived from human controls both stimulated with BBG and unstimulated in comparison with macrophages from patients with $\mathrm{HCC}$, where a significant increase was observed after 3 and 5 days of culture, however, without significant differences between stimulated and unstimulated cultures. In the study of Fan et al., glucan addition did not alter the basal ROS levels of murine macrophages (19). However, according to Vetvicka and Vetvickova, barley glucan insignificantly increased superoxide production in human HL-60 cells after $24 \mathrm{~h}$ of incubation (20). We observed significant changes during cultivation only in macrophages from patients with $\mathrm{HCC}$ but without differences between cultures treated or not with BBG.

In regard to superoxide anion generation in control rats, a significant response to BBG was detected only at maximal concentration $(20 \mu \mathrm{g} / \mathrm{ml})$ at the first measurement, and at 10 and $20 \mu \mathrm{g} / \mathrm{ml}$ at the second and third measurements. In DEN-treated rats, generation of superoxide anion significantly increased at the first time point only under the influence of $20 \mu \mathrm{g} / \mathrm{ml}$ of BBG. In the study of Fan et al. barley glucan did not change ROS generation during $48 \mathrm{~h}$ culture (19). On the other hand, in the report of Michałek et al. it was found that PGG-glucan stimulated increased respiratory burst in rat peritoneal and alveolar NR8383 macrophages cell line (15).

We established that during 5 days of culture, the concentrations of $\mathrm{BBG}$ that we used did not act cytotoxically, neither on human nor on rat macrophages. In 

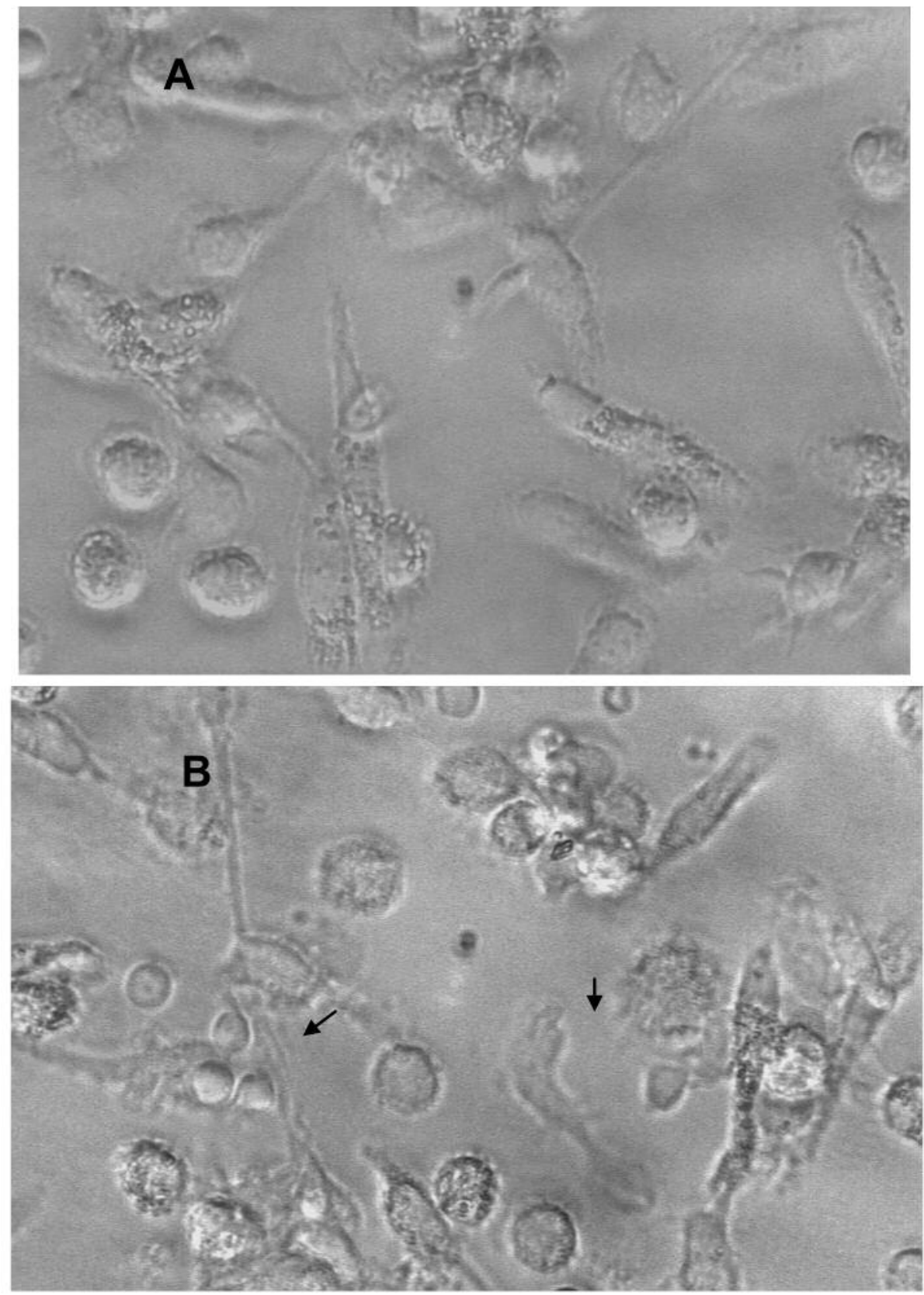

Figure 8. Phase-contrast micrography of monocyte-derived macrophage morphology from patients with HCC after 3 days of incubation without stimulation (A) and under the influence of $10 \mu \mathrm{g} / \mathrm{ml}$ of $\beta$-glucan. In unstimulated culture, morphologies included mostly rounded cells, and some spherical and elongated forms with long filopodia. In culture with $\beta$-glucan, there were a few rounded cells. Mostly, well-spread and elongated forms with long filopodia were seen. Arrows indicate microvilli present in activated forms of macrophages. Original magnification $\times 40$ (Olympus CK-40).

the study of Jang et al., BBG did not exert any cytotoxic effect on macrophages at concentrations of 1 to $100 \mu \mathrm{g} / \mathrm{ml}$ for $24 \mathrm{~h}$ (21). Chanput et al. also did not observe cytotoxicity of BBG at a concentration of $100 \mu \mathrm{g} / \mathrm{ml}$ on human monocytic leukemia cell line after up to 24-h incubation (22). In numerous animal models, $\beta$-glucans have shown broad anti-infective and antitumoral activities, including activation of macrophages, and no undesirable side-effects of BBG have yet been reported in humans and rats $(20,23)$.

Taking into account our results, we conclude that primary human and rats macrophages 'trained' in a BBG-containing microenvironment maintain a highly dynamic plasticity, 
which was clearly visible in cultures of macrophages from patients with HCC. These morphological changes of HCCderived macrophages take place together with their proinflammatory polarization, as reflected by ROS and RNI augmentation. In vitro models such as the one used in our study for macrophage polarization can be further explored in studies to investigate the influence of 'trained' human macrophages on isolated HCC cells in a quasi vivo cell culture system.

\section{Acknowledgements}

This study was supported by Grant no DEC-2014/15/B/Nz5/01587 from the National Science Centre, Poland.

\section{References}

1 Wang N, Liang HL and Zen K: Molecular mechanisms that influence the macrophage M1-M2 polarization balance. Front Immunol 5: 1-9, 2014.

2 Tomuleasa C, Soritau O, Rus-Ciuca D, Pop T, Todea D, Mosteanu O, Pintea B, Susman S, Kacso G and Irimie A: Isolation and characterization of hepatic cancer cells with stemlike properties from hepatocellular carcinoma. J Gastrointestin Liver Dis 19: 61-67, 2009.

3 Lee $\mathbf{J}$ and Lim KT: SJSZ glycoprotein (38 kDa) modulates macrophage type 1/2-related factors at hepatocarcinogenic stage in $N$-nitrosodiethylamine-treated Balb/c. Mol Cell Biochem 372: 17-26, 2013.

4 Wójcik M, Bobowiec R and Martelli F: Effect of carotenoids on in vitro proliferation and differentiation of oval cells during neoplastic and non-neoplastic liver injuries in rats. J Physiol Pharmacol Suppl 2: 203-213, 2008.

5 Borbath I and Horsmans Y: The role of PPAR $\gamma$ in hepatocellular carcinoma. PPAR Research 2008: 209520, 2008. doi:10.1155/ 2008/209520

6 Biswas SK, Sica A and Lewis CE: Plasticity of macrophage function by distinct molecular mechanisms. J Immunol 180: 2011-2017, 2008.

7 Saeed S, Quintin J, Kerstens HHD, Rao NA, Aghejenirefah A, Matarese F, Cheng SC, Ratter J, Berentsen K, van der Ent M, Sharifi N, Janssen-Megens EM, Huurne MT, Mandoli A, van Schaik T, Ng A, Burden F, Dowes K, Frontini M, Kumar V, Giammarellos-Bourboulis EJ, Ouwehand WH, van der Meer JWM, Joosten LAB, Wijmenga C, Martens JHA, Xavier R J, Logie C, Natea $M G$ and Stunnenberg HG: Epigenetic programming during monocyte to macrophage differentiation and trained innate immunity. Science 345: 1251086, 2014. doi: $10.1126 /$ science. 1251086

8 Tada R, Ikeda F, Aoki K, Yoshikawa M, Kato Y, Adachi Y, Tanioka A, Ishibashi K, Tsubaki K and Ohno N: Barley-derived $\beta$-D-glucan induces immunostimulation via a dectin-1-mediated pathway. Immunol Lett 123: 144-148, 2009.

9 Damoiseaux JGMC, Döpp EA, Beelen RHJ and Dijkstra CD: Rat bone marrow and monocyte cultures: influence of culture time and limphokines on the expression of macrophage differentiation antigens. J Leukocyte Biol 46: 246-253, 1989.
10 Ploeger DTA, Van Putten SM, Koerts JA, Van Luyn MJA and Harmsen MC: Human macrophages primed with angiogenic factors show dynamic plasticity, irrespective of extracellular matrix components. Immunobiology 217: 299-306, 2012.

11 Wessely-Szponder J, Bobowiec R and Szponder T: The influence of porcine prophenin on neutrophils isolated from rabbit blood during implantation of calcium sulphate graft material into bone tissue. World Rabbit Sci 20: 163-172, 2012.

12 Corraliza, IM, Campo, ML, Soler G and Modolell M: Determination of arginase activity in macrophages: a micromethod. J Immunol Methods 174: 231-235, 1994.

13 Wójcik M, Wessely-Szponder J and Kosior-Korzecka U: Proliferative and oxidative response of hepatocytes (Hep) and hepatic stellate cells (HSC) isolated from rats exposed to ketogenic diet. P J Vet Sci 17: 703-711, 2014.

14 Porta EA, Dytioco S, Aquino T, Dorado RD, Ooka S and Markell N: Evaluation of the effect of glucan on hepatic, renal and mammary tumorigenesis in rats. Res Commun Chem Pathol Pharmacol 52: 391-394, 1986.

15 Michałek M, Melican M, Brunke-Reese D, Langevin M, Lemerise K, Galbraith W, Patchen M and Mackin W: Activation of rat macrophages by Betafectin PGG-glucan requires crosslinking of membrane receptors distinct from complement receptor three (CR3). J Leukocyte Biol 64: 337-344,1988.

16 Geelhaar-Karsch A, Schinnerling K, Conrad K, Friebel J, Allers $\mathrm{K}$, Schneider T and Moos V: Evaluation of arginine metabolism for the analysis of M1/M2 macrophage activation in human clinical specimens. Inflamm Res 62: 865-869, 2013.

17 Arranz A, Doxaki C, Vergadi E, Dela Torre YM, Vapopridi K, Lagoudaki ED, Ieronymaki A, Venihaki M, Margioris AN, Stathopoulos EN, Tsichlis PN and Tsatsanis C: AKT1 and AKT2 protein kinases differentially contribute to macrophage polarization. Proc Natl Acad Sci USA 109: 9517-9522, 2012.

18 Campbell L, Emmerson E, Williams H, Saville CR, Krust A, Chambon P, Mace KA and Hardman MJ: Estrogen receptoralpha promotes alternative macrophage activation during cutaneous repair. J Invest Dermatol 134: 2447-2457, 2014.

19 Fan YG, Hu CW and Chy C: Effect of barley $\beta$-glucan on murine RAW264.7 macrophages against virulent Salmonella enteric serovar Typhimurium. Food Res Inter 45: 1106-1110, 2012.

20 Vetvicka V and Vetvickova J: Comparison of immunological effects of commercially available $\beta$-glucans. Appl Sci Reports 1:2, 2014. doi: 10.7243/2054-9903-1-2, available from http://www.hoajonline.com/journals/pdf/2054-9903-1-2.pdf

21 Jang AA, Park S, Lim JD, Kang SC, Yang KH, Pyo S and Sohl EH: The comparative immunomodulatory effects of $\beta$-glucans from yeast, bacteria, and mushroom on the function of macrophages. J Food Sci Natur 14: 102-108, 2009.

22 Chanput W, Reitsma M, Kleinjans L, Mes JJ, Sevelkoul HFJ and Wichers HJ: $\beta$-Glucans are involved in immune-modulation of THP-1 macrophages. Mol Nutr Food Res 56: 822-833, 2012.

23 Tanioka A, An WW, Kuge T, Tsubaki K and Nakaya K: Barley low molecular weight $\beta$-glucan potently induces maturation of dendric cells. Anticancer Res 31: 1647-1652, 2011.

Received September 6, 2016 Revised September 27, 2016 Accepted October 6, 2016 